\title{
A interação e o processo de negociação em L2
}

Cláudia Eliana Bassi

Deise Prina Dutra

Universidade Federal de Minas Gerais

Este trabalho apresenta uma investigação sobre a interação e as negociações desencadeadas por atividades comunicativas em uma sala de aula de nível básico de francês como segunda língua (L2). A partir da teoria sociointeracionista (VYGOTSKY, 1998), os resultados obtidos demonstraram que o processo interacional incentiva a troca de informações entre os aprendizes, favorecendo a negociação, e, também, que os aprendizes utilizaram estratégias de mediação para realizar as suas negociações. Os aprendizes receberam input modificado e tiveram mais oportunidades para a produção de output compreensível. Este relato, portanto, mostra que, ao negociar para a compreensão e a produção de textos em L2, os aprendizes se comprometeram, tornando-se agentes responsáveis e, sobretudo, colaboradores do processo de ensino/aprendizagem da L2.

This paper investigated the interaction and the negotiations generated by communicative activities proposed in a French as a second language (L2) basic class. Based on the socio-interactionist theory (VYGOTSKY, 1998), the results show that the interactional process encourages the exchange of information among learners, helping negotiation. Besides that, learners also use mediating strategies to do the negotiations. The learners receive modified input and have more opportunities to produce comprehensible output. This paper, therefore, shows that, to negotiate, to understand and to produce L2 texts, the learners committed themselves, becoming responsible agents, and, above all, collaborators in the L2 learning/teaching process.

No enfoque da análise interacional, várias investigações descrevem e analisam as interações verbais e não-verbais que ocorrem entre professores e aprendizes, e a estrutura de participação dos interactantes, assinalando a complexidade dos fatores envolvidos no processo interacional. O discurso em sala de aula de L2 é estudado, levando-se em conta as tomadas de turno, as interrupções de fala, o silêncio e os gestos dos interactantes (TSUI, 1995; ALLWRIGHT, 1980; CHAUDRON, 1988). As pesquisas revelam alguns papéis recorrentes do professor e do aprendiz na interação. O professor, em geral, atua como condutor 
e gerenciador do processo de aprendizagem e detém a maior parte dos turnos de fala (CAZDEN, 1988; TSUI, 1995; PAIVA, 1999). O aprendiz, habituado a essa aprendizagem centrada no professor, assume o papel receptivo e se esforça para atingir o objetivo traçado e, geralmente, assume a postura de que o sucesso de sua aprendizagem independe de uma relação interpessoal, ou seja, considera-se individualmente e não como membro de um grupo social. Segundo Stevahn, Bennett, Rolheiser (1995, p. 22), muitos alunos são induzidos a reflexões tais como "o fato de eu atingir o meu objetivo não está relacionado com o sucesso dos meus colegas". Muitos alunos também se comparam ao grupo e se preocupam em realizar o seu trabalho melhor do que o colega/ concorrente. Os mesmos autores consideram que alunos assumem posturas tais como "eu quero obter mais sucesso do que os meus colegas"; "eu vou ser o melhor" (STEVAHN, BENNETT, ROLHEISER, 1995, p. 22).

Ao buscar um novo paradigma para o ensino/aprendizagem das L2, as propostas educacionais atuais baseiam-se nos pressupostos da teoria sociointeracionista e da aprendizagem colaborativa. Seria aconselhável considerar o processo interacional como fator facilitador da aprendizagem, tendo em vista que viabiliza oportunidades efetivas para que os aprendizes compreendam e usem a língua-alvo. Propiciar ao aprendiz momentos de reflexão tais como "eu dividi informações com os colegas", "eu fui solidário com os membros do meu grupo", "eu consegui representar bem o meu papel no grupo" podem resultar em circunstâncias que facilitem a aquisição de conhecimento em L2 (STEVAHN, BENNETT, ROLHEISER, 1995, p. 33). ${ }^{1}$

A aprendizagem colaborativa é definida como uma forma de organização da aprendizagem que permite a grupos pequenos e heterogêneos de alunos atingir objetivos comuns de aprendizagem, apoiando-se em uma relação de interdependência, ou seja, a plena participação de todos no cumprimento de determinada tarefa (GAUDET et al., 1998). Nessa abordagem, o professor tende a atuar na sala de L2 como mediador do conhecimento lingüístico dos aprendizes e o caráter

\footnotetext{
${ }^{1}$ Não é objetivo deste artigo apresentar as reflexões dos alunos participantes da pesquisa. Um questionário individual composto de questões abertas em L1 possibilitou resgatar as suas percepções, uma vez que os alunos puderam expor o seu ponto de vista em relação ao trabalho realizado em sala e sua opinião sobre a aula colaborativa. Consultar Bassi (2002), para maiores detalhes.
} 
interativo da produção de conhecimento, ou seja, os modos de participação do outro nessa produção são ressaltados (GASS e VARONIS, 1985; PICA, YOUNG, DOUGHTY, 1987; PICA et al., 1991; PICA et al., 1996; DiCAMILLA e ANTÓN, 1997; SWAIN e LAPKIN, 1998).

O presente artigo apresenta os resultados da pesquisa que investigou a interação verbal como processo de negociação. Em torno desse tema, examinamos se a interação proporciona oportunidades para a aprendizagem de línguas e se a negociação entre os participantes é facilitada pelo uso de andaime como estrutura de apoio. Foi verificado que os aprendizes negociam as formas lingüísticas, os significados das mensagens e os conteúdos de textos em L2, no momento da execução de três tarefas comunicativas, isto é, o dictogloss, o jigsaw e o dictogloss com o jigsaw. ${ }^{2}$

Neste artigo, nossa exposição está subdividida em cinco partes. Inicialmente, apresentaremos os pressupostos da teoria sociointeracionista e suas implicações para o ensino/aprendizagem de uma L2. Na segunda parte, para abordarmos o processo de negociação, focalizaremos os seguintes tópicos: o foco de negociações, a seqüência de negociação e as estratégias mediadoras. Na terceira, é mostrada a base metodológica desta investigação, que se circunscreve nos parâmetros da pesquisa qualitativa. Na quarta, têm-se a análise e a discussão dos modos de participação dos alunos para negociar o significado, a forma e o conteúdo. ${ }^{3}$ $\mathrm{Na}$ conclusão, enfim, tecem-se considerações finais sobre a análise e o processo de investigação como um todo.

\section{A teoria sociointeracionista}

A teoria sociointeracionista, também conhecida como abordagem sociocultural, postula que o indivíduo se constitui e se desenvolve por intermédio de trocas estabelecidas com seu meio sociocultural. Nessa perspectiva, há o enfoque na socialização da aprendizagem, haja vista

\footnotetext{
${ }^{2}$ As tarefas comunicativas mencionadas neste artigo são analisadas em Bassi (2002).

${ }^{3}$ Os episódios de negociação discutidos neste artigo serão analisados tal como foram expressos pelos alunos participantes da investigação, ou seja, as falas são apresentadas tal qual foram produzidas, sem adequação às normas gramaticais da língua francesa, devido ao objetivo de se investigarem as interações ocorridas entre os alunos, tornando-se fundamental a transposição exata de suas negociações.
} 
que o processo de construção do conhecimento baseia-se em experiências interativamente criadas. O background cultural, lingüístico e o conhecimento de mundo integram-se em uma relação de compartilhamento e de negociação, tendo em vista o valor potencial da interação para o desenvolvimento sociolingüístico do aluno.

O pressuposto da interação como um processo co-construído é a base teórica norteadora da nossa reflexão sobre os modos efetivos de participação do outro na compreensão e produção de textos em L2. Em articulação com essa visão de interação, em nossa pesquisa, são adotadas as reflexões de Vygotsky (1998) sobre o papel da mediação do outro no processo de desenvolvimento sociocognitivo do aluno do ensino fundamental. A mediação, na teoria vygotskyana, é de fundamental importância, visto que é por meio desse processo que as funções psicológicas humanas se desenvolvem.

Segundo Vygotsky (1998), a interação social é tida como contexto e origem do desenvolvimento mental do indivíduo e todo desenvolvimento cognitivo é interpsicológico, ou seja, surge como resultado da interação entre os indivíduos (WERTSCH, 1991). Tal visão é compartilhada por Donato (1996, p. 7), para quem "aprender é uma forma de socialização entre indivíduos e não somente um processo de recebimento de input em um cérebro isolado.” "Assim sendo, para ocorrer a internalização do conhecimento, deve haver um movimento de passagem de ações realizadas no plano social (o aluno com seus pares, processo interpessoal) para as ações internas (o aluno consigo mesmo, portanto, um processo intrapessoal). Em seus estudos, Vygotsky destaca que as experiências socioculturais funcionam como mediadoras dos processos mentais do indivíduo. O apoio do outro faz com que o indivíduo trabalhe na ZDP (zona de desenvolvimento proximal), definida como a diferença entre o que o indivíduo é capaz de realizar quando age só e o que é capaz de realizar contando com o apoio de outra pessoa (VYGOTSKY, 1998).

Os sujeitos, em interação, constróem seus enunciados na dinâmica de sua elaboração, no movimento de produção e construção conjunta em negociação. Para descrever esse movimento interlocutivo e interativo, em nossa pesquisa, o construto de andaime foi adotado em

4 “...learning is a form of socialization between individuals and not merely inputprocessing carried out within an isolated brain."(DONATO, 1996, p. 7) 
simetria com a teoria desenvolvimentista vygotskyana. O construto de andaime, definido como a participação efetiva do outro, foi inicialmente proposto em pesquisas de aquisição naturalística em L1 (WOOD; BRUNER; ROSS, 1976, citado por WELLS, 1998), passando a ser desenvolvido pelos sociointeracionistas em estudos sobre interação em sala de aula como contexto de aprendizagem/construção do conhecimento.

Abordando o contexto da sala de aula, vários autores utilizaramse da metáfora de andaime para demonstrar como o par mais experiente (o tutor, no caso, o professor ou o falante nativo) auxilia o menos experiente (o aluno ou o falante não-nativo) a executar uma tarefa que estaria além de suas potencialidades por meio da construção interativa do conhecimento (FREEMAN, 1992; PICA, 1987; PICA et al., 1989). Entretanto, outro padrão de análise foi amplamente divulgado em estudos sobre mediação em sala de aula. DiCamilla e Antón (1997), Donato (1994), Swain e Lapkin (1998), Klinger e Vaughn (2000) demostraram que a mediação do outro ocorre também entre alunos de competência equivalente que trabalham em pares. Assim, o trabalho colaborativo propicia o andaime entre pares com conhecimento homogêneo ou heterogêneo, o que pode ser fundamental para o desenvolvimento lingüístico dos aprendizes que o utilizam.

Nessa perspectiva, muitos pesquisadores apontam que é preciso enfatizar, em sala de aula de L2, o papel desempenhado pelo aluno como co-produtor para a aquisição de conhecimento (FORMAN e CAZDEN, 1985; DONATO e LANTOLF, 1990; TUDGE, 1990; PICA et al., 1996; KLINGNER e VAUGHN, 2000). Nesses trabalhos, os pesquisadores salientam a importância da colaboração entre pares aprendizes (peer tutoring e peer collaboration), por entender que os trabalhos em dupla ou em grupos geram situações com potencial de aprendizagem que não devem ser ignoradas pelos professores.

As teorias de aprendizagem de L2 em contexto sociocultural concentraram-se, inicialmente, em estabelecer uma relação processual entre compreensão, produção e aquisição de línguas. Adicionalmente, a participação dos aprendizes na interação proporcionaria melhor compreensão e mais oportunidades de produção da L2 (PICA et al., 1991). Nesse contexto de discussão, hipóteses teóricas foram formuladas e se destacaram na literatura pela relevância de suas perspectivas: a hipótese de interação (LONG, 1983) e a hipótese de output compreensível (SWAIN, 1985, 1995a,b). 
Para que se compreenda a valorização do papel do output na aquisição da L2, é necessário destacar, primeiramente, a hipótese de input compreensível, desenvolvida anteriormente às propostas socioculturais. Segundo Krashen (1983), para adquirir conhecimento, basta que o aprendiz esteja exposto à L2, pois compreender o input leva necessariamente à aquisição da L2. Além disso, em sua visão, o input recebido deve estar um nível acima da competência em L2 do aprendiz. Dessa forma, o input compreensível seria $i+1$, sendo o $i$ a competência atual do aprendiz na língua. Todavia, a exposição à língua-alvo não é suficiente para a sua aquisição. Para reconhecer e interiorizar a L2, o aprendiz deve compreender o significado da mensagem e de estruturas da L2 (LONG, 1983). Para compreender, a interação negociada deve ocorrer e, assim, a aquisição pode ser favorecida. Durante a negociação, os aprendizes podem alcançar a compreensão mútua por meio de modificações de seu output (LONG, 1996). Assim sendo, pesquisadores discutem que os aprendizes devem ter oportunidades para manipular e estruturar a sua produção, trabalhando o output (SWAIN, 1985; PICA et al., 1991). As experiências com a produção de L2 conforme foram descritas por Swain (1985, 1995a, b, 1998 e 2000) ajudam os aprendizes a perceber melhor sua interlíngua (IL), ${ }^{5}$ o que gera a internalização de formas e estruturas da L2. Dessa forma, os momentos de produção de output são considerados importantes para que os aprendizes possam, por meio do uso da IL, adquirir conhecimento nesse processo.

A partir da hipótese de interação, as pesquisas direcionaram-se para operacionalizar o conceito de negociação como um fator central para aquisição da L2 (GASS e VARONIS, 1985 e 1994; PICA; YOUNG; DOUGHTY, 1987; PICA et al., 1991; PICA, 1994). Vários estudos mostraram que as modificações interacionais promovidas por negociação podem ter um efeito positivo na qualidade da produção dos aprendizes e favorecer a aquisição da L2.

Segundo a definição de Pica (1994), negociação é uma forma de interação utilizada para que os alunos negociem o input e explorem seu output. A negociação ocorre durante a interação quando um interlocutor emite sinais de que não obteve input compreensível (PICA

\footnotetext{
${ }^{5}$ Termo proposto por Selinker (1972), compreendido como a língua de transição dos aprendizes entre a língua nativa e a língua-alvo.
} 
et al., 1996). Desse modo, o aluno poderá solicitar ao seu interlocutor uma explicação sobre algo que não compreende ou não está claro na sua fala, viabilizando, portanto, o prosseguimento da produção conjunta. O outro, então, responde, freqüentemente repetindo ou modificando seu output. A versão modificada pode ser uma palavra ou frase segmentada da fala inicial, uma paráfrase ou uma substituição com sinônimos (PICA et al., 1996). Por meio da negociação resultante, os alunos têm oportunidades para compreender e usar a língua. Adicionalmente, eles podem receber input compreensível e ter mais oportunidades para a produção de output. Swain (1985, 1995a, b) cunhou o termo output compreensível para trabalhar nessa perspectiva. Basicamente, ela defende a visão de que o aluno se esforça para alcançar a compreensão, ao produzir, ao ser impelido a dar uma resposta para o seu interlocutor e ao testar novas formas lingüísticas ou modificá-las. Dessa maneira, o aluno não compreende apenas recebendo o input de seu interlocutor, mas produzindo output.

Para explorar o papel do output, Swain e Lapkin (1995, 1998) discutem o que elas chamam de diálogos colaborativos, observados em termos de Language Related Episodes - LREs - ou seja, episódios de negociação relacionados com a língua em estudo. Nessas conversações, os alunos analisam tanto a língua quanto a mensagem de seus textos. Em nossa pesquisa, analisamos os LREs como uma fonte de aprendizagem do francês como L2.

Neste artigo, a fim de analisarmos alguns LREs, definiremos o foco de negociação e apresentaremos brevemente como uma seqüência de negociação pode ser desencadeada durante um processo interacional. A análise descritiva apresentada tratará a natureza da interação e o uso de andaime como estratégia mediadora para negociar o significado (PICA et al., 1989), a forma lingüística (LYSTER e RANTA, 1997) e o conteúdo (VAN DEN BRANDEN, 1997).

\section{O processo de negociação}

\section{O foco de negociações}

Considerando a relevância do papel da negociação para a aquisição da L2, apresentaremos uma distinção entre os tipos de negociação que podem ocorrer durante a interação. 
Pica (1994) realça o efeito positivo da negociação para o processo de aquisição: a negociação é uma ajuda para compreensão do significado da L2. Pica et al. (1989, p. 65) caracterizam a negociação de significado como as "trocas entre os alunos e seus interlocutores para solucionar problemas de comunicação e para atingir a compreensão mútua." ${ }^{6}$ Assim sendo, os interlocutores trabalham no nível do significado da mensagem e se empenham para alcançar a compreensão de palavras e idéias expressas pelo outro. Durante a negociação de significado, o fluxo conversacional está no nível semântico, tendo em vista que tal negociação lida com problemas de compreensão da mensagem.

Já alguns estudiosos sugerem a importância de um enfoque diferenciado para outros tipos de negociações encontradas durante o processo interacional (SWAIN, 1985; 1995b; 2000; VAN DEN BRANDEN, 1997; LYSTER e RANTA, 1997). A negociação pode focalizar, também, a forma lingüistica, uma vez que ela "não enfatiza somente o significado da mensagem, mas fornece também oportunidade para focar na forma da mensagem" (PICA, 1994, p. 513). ${ }^{7}$

Van den Branden (1997) trata, também, da negociação sobre o significado e sobre a forma, mas estende a noção de negociação e inclui a negociação sobre o conteúdo. Durante a negociação de conteúdo, o interlocutor conduz a negociação para o nível do conteúdo da conversação, ao demandar mais informações para o melhor entendimento do assunto tratado. Para Van den Branden (1997, p. 605), a negociação de conteúdo é a "continuação da interação visando forçar aquele que descreve a prover mais informação do que as oferecidas espontaneamente na descrição." O autor observa, ainda, que a "negociação de conteúdo difere da negociação de forma e de sentido, pois não constitui um desvio do

\footnotetext{
6 "...exchanges between learners and their interlocutors as they attempt to resolve communication breakdowns and to work toward mutual comprehension." (PICA et al., 1989, p. 65)

7 "...negotiation as a process not only emphasises communication of message meaning, it also provides an opportunity to focus on message form." (PICA, 1994, p. 513)

8 "...stretches of interaction aimed at pushing the describer to provide more information than spontaneously offered in the description." (VAN DEN BRANDEN, 1997, p. 605)
} 
fluxo principal da conversação" (VAN DEN BRANDEN, 1997, p. 594). ${ }^{9}$ Dessa forma, os interlocutores conduzem a negociação para o conteúdo quando necessitam complementar a idéia expressa.

\section{A seqüência de negociação}

O modelo interacionista proposto e desenvolvido por Varonis e Gass (1985) e Pica et al. (1989, 1991) estabelece que uma seqüência completa de negociação, seja de significado, de forma ou de conteúdo, deve compreender quatro categorias de enunciados.

Para ocorrer uma negociação, o primeiro enunciado, o gerador da negociação $(G)$, não é compreendido ou não é aceito pelo interlocutor. Entende-se como gerador da negociação $(G)$ uma expressão, uma palavra ou qualquer informação lingüística que, ao não ser compreendida, ocasiona o início do processo de negociação. Na categoria dos sinais de não compreensão (SNC), destacam-se os sinais emitidos pelo interlocutor para mostrar ao outro que a sua fala não foi compreendida. Em seguida, o interlocutor, ao considerar o sinal de não compreensão, produz uma resposta imediata (R). Na categoria dos sinais de compreensão (SC), encontram-se os sinais emitidos pelo interlocutor para indicar o entendimento do LRE, o prosseguimento da conversação e o conseqüente fechamento da seqüência de negociação. Uma seqüência de negociação acontece sempre quando os interlocutores em interação emitem sinais de não entendimento mútuo.

\section{As estratégias mediadoras}

Analisando interações entre aprendizes de uma L2, estudos que se baseiam na teoria sociointeracionista (FORMAN e CAZDEN, 1985; GASS e SELINKER, 1994; PICA, 1994; ELLIS, 1999; DONATO, 2000; SWAIN, 2000; FIGUEIREDO, 2001) afirmam que os alunos utilizam determinadas estratégias mediadoras para negociar e, assim, facilitar o cumprimento de atividades propostas em sala, favorecendo o desenvolvimento lingüístico. As três estratégias mediadoras são as

\footnotetext{
9 "Negotiation of content differs from negotiation of form or meaning in that it does not constitute a side-sequence to the main flow of conversation." (VAN DEN BRANDEN, 1997, p. 594)
} 
fontes externas, o uso da L1 e os andaimes. Neste artigo, analisaremos brevemente os andaimes utilizados em interação.

Vários autores apresentam taxonomias para os tipos de andaimes que podem ser aplicados durante a negociação (LONG, 1983; SWAIN, 1985; VARONIS e GASS, 1985; PICA, 1987; PICA et al., 1989; LYSTER e RANTA, 1997; VAN DEN BRANDEN, 1997; SWAIN e LAPKIN, 1998).

Segundo Long (1983), quando alguma dificuldade de compreensão impede o entendimento da conversação, certas estratégias são utilizadas pelo aluno na tentativa de reparar e modificar a estrutura dos enunciados: os pedidos de esclarecimento, os pedidos de confirmação e as checagens ou verificação de compreensão. Baseando-nos no trabalho proposto por Lyster e Ranta (1997), neste estudo os participantes utilizaram dois tipos de feedback em sua tentativa de sinalizar o não entendimento do enunciado: a reformulação e o feedback metalingüístico. Dessa forma, nossa proposta é que os sinais de não compreensão (SNC) podem se subdividir em pedido de esclarecimento (LONG, 1983), feedback metalingüistico (LYSTER e RANTA, 1997), pedido de confirmação (LONG, 1983) e reformulação (LYSTER e RANTA, 1997).

Em nosso estudo, analisamos diferentes tipos de respostas que o aluno pode oferecer ao colega, sempre buscando o entendimento mútuo para executar as tarefas: a repetição da fala do outro e a auto-reformulação (LYSTER e RANTA, 1997). Além disso, há como respostas a estratégia de avoidance (SCHMIDT e FROTA, 1986). Segundo os autores, a estratégia de avoidance é utilizada quando o interlocutor evita o que considera uma provável inadequação e opta por formular algo que considera mais preciso. Em outras palavras, avoidance é uma estratégia para evitar o erro. Em resumo, em nosso estudo, serão analisadas quatro tipos de respostas (R) emitidas pelos interlocutores durante as negociações: verificação de compreensão (LONG, 1983), repetição da fala do outro e auto-reformulação (LYSTER e RANTA, 1997) e estratégia de avoidance (SCHMIDT e FROTA, 1986). Por fim, utilizamos também os termos sinais de compreensão (SC) como sinal de entendimento do LRE e sinal de continuação do LRE (PICA et al., 1989).

A figura 1 propõe-se a integrar o seqüenciamento das negociações (de significado, de forma e de conteúdo) e a utilização de andaime como estratégia mediadora para favorecer a execução das atividades comunicativas propostas. Segundo a figura, o gerador da negociação implica o uso de andaimes e podemos verificar a grande gama de 
estratégias mediadoras que foram usadas durante as atividades comunicativas. Dessa forma, o gerador não se coloca no mesmo nível dos sinais de não compreensão, das respostas e dos sinais de compreensão.

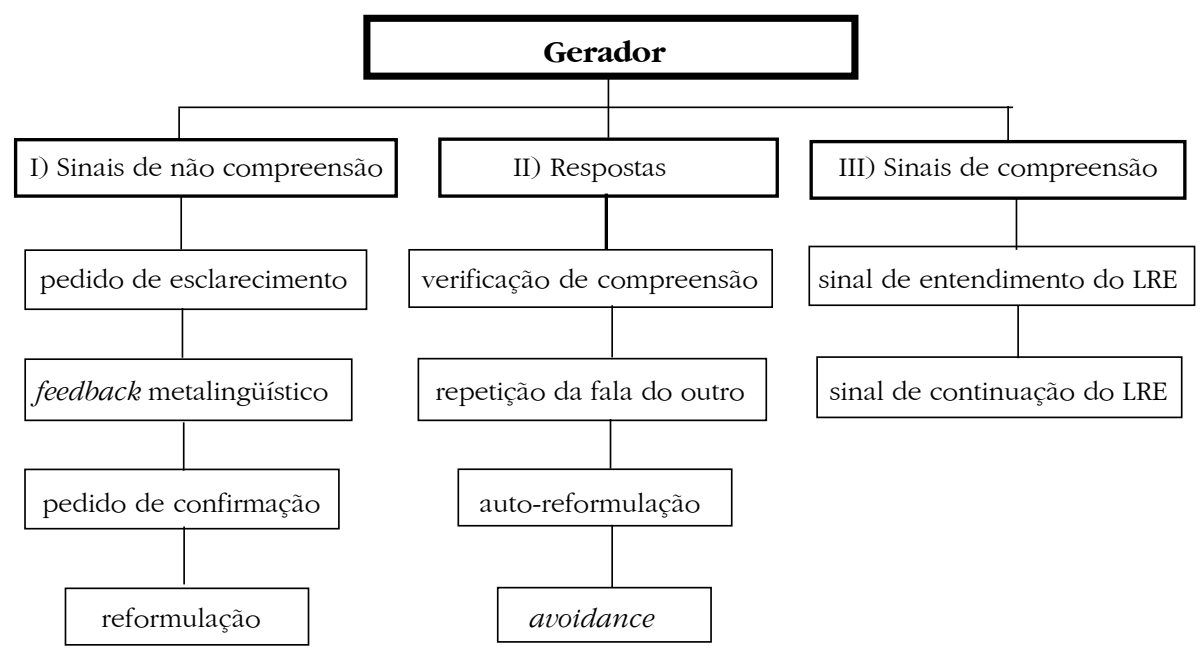

FIGURA 1 - Seqüência de negociação e uso de andaime durante as atividades

\section{Metodologia}

A pesquisa foi realizada na Escola Fundamental do Centro Pedagógico (EFCP) da Universidade Federal de Minas Gerais (UFMG) durante o primeiro semestre letivo de 2001. Foram investigados nove participantes em sala de aula e os próprios alunos escolheram os seus pseudônimos: Anna, Isabella, Gabriel, Paula, Enaira, Ana Carolina, João, Adriana e Juliana. Todavia, neste artigo apresentamos dados de quatro dos participantes, com o intuito de ilustrar interações representativas do grupo. O processo de aprendizagem desses participantes é particular, mas a análise apresentada pode tornar-se útil também a outras turmas de aprendizes de uma L2.

Em nossa investigação, interessou-nos pesquisar o processo de aprendizagem do francês como L2 entre alunos do ensino fundamental (7ํ e $8^{\circ}$ anos escolares). Para identificar a presença de negociação e descrever o processo de produção de output compreensível (SWAIN, 
1985), objetivo da pesquisa, optou-se pela realização de uma pesquisa dentro do paradigma das pesquisas qualitativas para análise de um estudo de caso. Foram usados instrumentos como questionários e gravações em áudio e vídeo, o que favoreceu uma melhor análise da interação e da negociação entre os alunos e do papel das mesmas no processo de aprendizagem da L2.

A pesquisa realizada configura-se como uma observação participativa, pois a primeira autora deste artigo era também a professora da turma e interagia mediando o conhecimento dos alunos. Foram criadas as atividades a serem exploradas em sala e os alunos foram monitorados. Aplicamos três tarefas comunicativas, o dictogloss, o jigsaw e o dictogloss com o jigsaw. Essas atividades são utilizadas colaborativamente em sala de aula para favorecer mais oportunidades de aprendizagem da L2. Segundo Swain e Lapkin (1998 e 2001), elas encorajam o aprendiz a refletir principalmente sobre o output produzido em negociação, o que pode ser uma importante fonte de aprendizagem da L2.

Segundo Swain (1998), o dictogloss propõe-se a incentivar os alunos a discutir sobre a L2 que estão reconstruindo e a formular e testar suas hipóteses para a produção de um texto escrito. A premissa dessa atividade é de que o aluno deve recriar, por escrito, com seu par, um texto lido pelo professor em ritmo normal e sem interrupções. No jigsaw, os alunos devem compor uma história baseada em uma série de imagens seqüenciadas. Dessa forma, cada participante detém informações necessárias para que o par/grupo finalize a atividade (SWAIN e LAPKIN, 1998, 2001).

Optou-se por aplicar, em sala, textos e imagens preparados especialmente para as atividades coletadas, pois visava-se garantir que os participantes recebessem input focado (input enhancement) em um certo contexto e em determinadas estruturas da língua-alvo (LIGHTBOWN e SPADA, 1990; WHITE, 1998). O foco foi na descrição de pessoas e em ações quotidianas, pois os alunos precisavam de mais oportunidades para receber input e produzir output com estruturas que expressassem essas idéias.

\section{Análise de dados}

Os alunos participantes deste estudo têm entre si certa familiaridade, tendo em vista que estudam juntos há vários anos. Durante a interação, 
várias trocas foram realizadas com o intuito de cumprir as atividades demandadas e outras, não. Assim sendo, inicialmente distinguimos sobre o que conversavam durante as interações, ou seja, analisamos o foco das negociações. O passo seguinte de nossa investigação foi averiguar como os alunos interagiam, ou seja, examinar o uso de andaime como estratégia mediadora para negociar o significado, a forma e o conteúdo.

\title{
Sinais de não compreensão
}

No LRE 1, o pedido de esclarecimento de Isabella sinaliza a não compreensão do significado do enunciado que foi gerado, pois a aluna sinaliza algum problema na compreensão da mensagem. Gabriel está ciente de que sua construção é incompreensível e usa a L1 para justificar:

\author{
Gabriel: elle (+) elle (+) une douche \\ $>$ Isabella: não entendi \\ Gabriel: é porque tá faltando uma palavra/ ela tá tomando banho \\ Isabella: ah' elle prend une douche \\ Gabriel: isso mesmo
}

(LRE 1)

Para sinalizar ao colega que sua produção não foi compreendida, o aluno, apoiando-se no seu conhecimento metalingüístico da L2, refuta a formulação que lhe foi sugerida sem, contudo, explicitar a forma mais adequada, mas comenta, informa ou pergunta sobre a proposta em discussão. No LRE 2, Isabella faz uma pergunta explícita e verbaliza seu problema lingüístico. No seu turno, Gabriel gera e testa hipóteses para o artigo mais adequado para o item noir. Isabella, baseando-se no seu conhecimento, rejeita a forma lexical proposta e usa a L1 para comentar a inadequação. O feedback metalingüístico recebido faz com que Gabriel se lembre do item lexical adequado e auto-reformule a proposição, gerando, assim, nova proposta que responde ao sinal inicial de Isabella:

Isabella: legal 'vamos formar uma nova frase' ele adora velas para uma noite romântica ((risos)) noite é masculino ou feminino"

Gabriel: noir" le noir/ não/ acho que é la noir

$>$ Isabella: mas nãoé noir noir épreto

Gabriel: ah é (+) é nuit la nuit

(LRE 2) 
Para sinalizar com um pedido de confirmação, o aluno repete parte ou toda a fala do colega para checar o que foi dito. No episódio 3, Gabriel repete parte da fala de sua colega para confirmar o que ouviu. Isabella infere, no pedido de confirmação de seu par, o desconhecimento do significado do verbo prendre (elle prend). Assim sendo, ela torna seu output compreensível, utilizando a L1:

Isabella: elleprendune voiture

$>$ Gabriel: prend"

Isabella: é prend, pegar tomar, entendeu" elle prend une voiture Gabriel: elle prend une voiture no meu também

(LRE 3)

O sinal de não compreensão pode surgir como uma reformulação do que foi proposto. No episódio 4, Paula reformula o enunciado, sugerindo o emprego da palavra douche, pois acredita ser mais adequado:

Ana: $\quad$ depuis Cécile $(+)$ depuis elle

Paula: [depuis elle

Ana: $\quad$ prend un bain

$>$ Paula: ou une douche

Ana: não, un bain/ na banheira é bain, no chuveiro, rapidinho, é douche/ aqui ela não precisa economizar energia

(LRE 4)

Os quatro tipos de sinais de não compreensão produzidos pelos alunos participantes da pesquisa demonstram que eles usam estratégias variadas para atingir o objetivo comum de completar a atividade proposta. Após emitirem os sinais de não compreensão, os alunos recebem de seus parceiros uma resposta ou explicação, deixando-os mais seguros para futuras interações.

\section{Respostas}

A verificação de compreensão ocorre quando o aluno confere se o colega compreendeu e se concorda com a sua produção. Pode-se verificar que existe preocupação com o entendimento do colega, ou seja, os alunos demonstram engajamento com o processo de produção em conjunto e com o compartilhamento de conhecimento. Assim, no LRE 5, Isabella demonstra internalização de conhecimento e verifica se o colega compreendeu a explicação: 
Gabriel: ilprend le petit déjeneur et je lis le journal

Isabella: je reste à la table pour lire le journal

Gabriel: je resté

$>$ Isabella: je reste, quer dizer que ele fica lendo o jornal dele enquanto toma café, entendeu"

Gabriel: entendi

(LRE 5)

O aluno pode repetir a sugestão oferecida pelo colega para internalizar algo que não sabe ou consolidar algo que esqueceu. No episódio abaixo (LRE 6), Anna sinaliza em L1 o seu entendimento inadequado do verbo se coucher. Portanto, ela necessita repetir a oração formulada por sua colega para internalizar o conhecimento:

Anna: a 11, deixa eu ver o que é (+) il vê télé/ eu consegui pegar isso/ vê como que você põe (+) a 11, é assim, ele chega do trabalho e vai pra televisão $(++)$ então a 15 vai ser que ele/ je joue ao football (++) a 16, je vais ao cinéma/ a 17, peraí/ você está em qual" na 13' tem que ver como que fala né' você sabe"

Paula: ilsecouche

Anna: não, ele SE dorme"

Paula: ele se deita

$>$ Anna: ab tá (+)ilse couche

(LRE 6)

Os alunos podem, também, auto-reformular as suas construções, a partir da contribuição do colega, para produzir o output com mais adequação. No episódio 7, observa-se que o pedido de confirmação feito por Gabriel em L1 gera uma auto-reformulação de sua colega e conduz à produção de output compreensível. Após a auto-reformulação de Isabella, há a recuperação do que ela havia dito anteriormente (il se lève), que funciona como um andaime para a sentença seguinte de Gabriel:

Gabriel: vai, você começa

Isabella: il se lève à 6 heures

Gabriel: ele se desperta"

> Isabella: não desculpa, il se réveille à 6 beures

Gabriel: então (+) il se lève à six heures vingt minutes

(LRE 7) 
No LRE 8, Isabella não se convence com as respostas de Gabriel em relação ao artigo que deveria acompanhar o vocábulo journal. Dessa forma, ela prefere optar por uma forma mais segura e usa o adjetivo possessivo como uma estrutura alternativa (son journal). Neste exemplo, Isabella utiliza a estratégia de avoidance ao preferir uma forma já conhecida, rejeitando uma proposta devido ao seu não convencimento:

Isabella: é lê jornal só" (+) lire journat' tá errado

Gabriel: lire le journal não dá não" põe le

Isabella: adoro a sua idéia (+) ele gosta de velas' isso é maravilhoso

Gabriel: põe le journal

Isabella: journal é feminino ou masculino"

Gabriel: é igual em português mesmo/le journal/.../

Isabella: eu escrevi isso naquele texto /.../

Gabriel: naquele texto do horóscopo (++) eu acho que é le (+) você gosta de ler' ler o quê" le journal

$>$ Isabella: eu não gosto de ler/ isso não vem ao caso, isso não vem ao caso (+) põe son journal son journal fica melhor

(LRE 8)

Percebemos, acima, quatro tipos de respostas para solucionar problemas de compreensão de um dos interlocutores. Essas respostas evidenciam estratégias como: verificação de compreensão, repetição de oração formulada, auto-reformulação e avoidance.

\section{Sinais de compreensão}

Utilizando o sinal de entendimento do LRE, o aluno demonstra que entendeu a seqüência negociada. No LRE 9, Isabella parte das hipóteses feitas por seu colega, a fim de refletir sobre o artigo apropriado. Gabriel sinaliza que compreende a associação sugerida por sua colega e repete o que foi proposto por Isabella, usando le samedi como andaime para a sua próxima intervenção:

Gabriel: é au samedi no sábado"

Isabella: au samedi"

Gabriel: en samedi'le samedi'

Isabella: deve ser le samedi (+) se é le week-end, é le samedi é a mesma coisa

$>$ Gabriel: tá, LE samedi je vais joue /.../

(LRE 9) 
Utilizando um sinal de continuação, o aluno indica a sua intenção de buscar outro LRE. No episódio 10, Isabella muda duas vezes o foco de negociação e propõe novo LRE, após sinalizar o entendimento da seqüência negociada:

Gabriel: ilporte costume
Isabella: é une costume"
Gabriel: le costume ou la costumé une costume un costume'le
costume é un costume né'
Isabella: mhm mhm (+) e manteau"
Gabriel: un manteau
Isabella: pronto (++) e agora' que mais que tem no desenhinho
(LRE 10) nós não colocamos"

Os LREs que exemplificam os sinais de compreensão são de dois tipos: sinal de compreensão e sinal de continuação. Esses sinais possibilitam que o par/grupo, confiante na compreensão de seus membros, possa prosseguir e completar a atividade.

Nos 10 LREs analisados, como verificamos, os alunos enfatizaram um tipo de negociação mais do que o outro, ora a forma (por exemplo, LREs 6, 8 e 10), ora o significado (por exemplo, LREs 1, 3 e 5) ou o conteúdo (por exemplo, LRE 4). Entretanto, observamos que os alunos também negociaram o significado, a forma e o conteúdo simultaneamente. Iniciando o LRE com o foco na forma lingüística, o aluno sinalizou a necessidade de compreensão do significado (LRE 2). Verificamos, também, que a negociação entre os participantes foi facilitada pelo uso de andaime como estrutura de apoio (LREs 7 e 9).

\section{Considerações finais}

A pesquisa realizada teve como objeto de investigação a análise das interações verbais observadas entre nove alunos do ensino fundamental em seu processo de aquisição/aprendizagem do francês como L2. Como foi discutido neste artigo, as pesquisas com base sociointeracionista argumentam que determinadas tarefas comunicativas podem favorecer a aquisição da L2 por meio da negociação de significado e de forma (SWAIN e LAPKIN, 2001) e de conteúdo, como discutido por Van den Branden (1997). Como os dados de nossa pesquisa revelaram, para executarem três tarefas comunicativas (o dictogloss, o 
jigsaw e o dictogloss com o jigsaw), os alunos se mostraram propensos a compartilhar e a socializar o seu conhecimento e utilizaram, para tal fim, a mediação do outro para co-construir conhecimento em L2. As atividades em questão ratificam a visão dos teóricos sociointeracionistas sobre o compartilhamento de conhecimento e corroboram a hipótese de output compreensível (SWAIN, 1985 e 1995a, b).

Segundo os resultados de nossa pesquisa, tarefas como o dictogloss e o jigsaw favorecem negociações, seja de significado, de forma e/ou de conteúdo, e servem como um contexto para prover o aluno com input e output compreensíveis. Como os dados revelaram, a fim de produzir um texto escrito, ${ }^{10}$ os alunos investiram nos três tipos de negociação, gerenciando a sua quantidade conforme a necessidade do grupo.

Em relação ao uso de andaime como estratégia mediadora, os sinais de não compreensão e as respostas observados validam a hipótese de output compreensível (SWAIN, 1985 e 1995a, b). Observou-se que, no dictogloss e no jigsaw, os alunos optaram por questionar o input recebido e se motivaram para tornar o output mais compreensível. Conforme podemos verificar, após iniciarem a conversação, os participantes emitiram sinais de não entendimento e tiveram a oportunidade de negociar com seus parceiros, respondendo de várias maneiras aos seus interlocutores, com o objetivo de produzir e tornar o output compreensível. Assim sendo, a adoção de estratégias mediadoras combinadas durante a interação gerou output compreensível, o que vem demonstrar a relevância do papel da negociação para a aquisição da L2.

O uso de andaime como estrutura de apoio e estratégia de mediação vem reiterar a importância da teoria sociointeracionista para a aprendizagem das L2. Em nosso estudo, os alunos do ensino fundamental estão aptos a conduzir o parceiro a modificar sua produção lingüística por meio de negociações. Como foi analisado neste artigo, os alunos puderam se questionar e tiveram oportunidades reais para discutir e aplicar suas hipóteses para solucionar problemas lingüísticos. Constatou-se que alunos pouco proficientes em L2 são capazes de

\footnotetext{
${ }^{10}$ Este artigo apresentou somente as interações verbais entre os alunos geradas a partir da proposta de atividades comunicativas. Apesar de os alunos terem produzido textos escritos, não foi o foco deste artigo analisar esses textos.
} 
modificar o input e o output via negociação e troca de informação e se colocam empenhados para produzir output compreensível durante a interação. Nesse contexto, verificamos que a seqüência de negociação criou a malha interacional durante as atividades comunicativas, e a exposição de idéias e construções foi enfrentada pelos participantes de forma colaborativa e produtiva.

Os resultados obtidos em nossa pesquisa revelaram os efeitos potenciais das negociações para a compreensão e produção de textos em L2, como também ofereceram evidência empírica para os efeitos da negociação na produção de output dos alunos. Assim sendo, podemos afirmar que a negociação desempenhou papel fundamental para a compreensão e produção de textos em L2.

Dessa forma, nosso estudo pretende contribuir para uma melhor compreensão sobre o papel da negociação na aprendizagem da L2. Espera-se que, com o reconhecimento da interação como uma oportunidade para reflexão sobre a língua e uma oportunidade para a troca de experiências sociolingüísticas entre aprendizes, os padrões tradicionais de ensino/aprendizagem de uma L2 e os papéis do aluno receptivo e do professor como centralizador da aprendizagem sejam repensados e reconsiderados. Como foi demonstrado neste artigo, os alunos podem ser agentes colaboradores para construir conhecimento lingüístico, o que poderá muito favorecer a sua aprendizagem.

\section{Referências Bibliográficas}

ALLWRIGHT, D. Turns, topics and tasks. In: LARSEN-FREEMAN, D. (Ed.). Discourse analysis in second language research. Rowley: Newbury, 1980.

BASSI, C.E. O efeito de negociações: a co-construção de conhecimento em sala de aula de francês como L2. 2002. 208 f. Dissertação (Mestrado em Lingüística Aplicada ao Ensino de Línguas Estrangeiras) - Faculdade de Letras, Universidade Federal de Minas Gerais, Belo Horizonte.

CHAUDRON, C. Second language classrooms. New York: Cambridge Univ. Press, 1988.

CAZDEN, C. B. Classroom discourse. Portsmouth: Heinemann, 1988. p. $159-96$. 
DiCAMILLA, F. J.; ANTÓN, M. Repetition in the collaborative discourse of L2 learners. The Canadian Modern Language Review, v. 53, n. 4, p. 60929, June 1997.

DONATO, R. Collective scaffolding in second language learning. In: LANTOLF, J. P.; APPEL, G. (Ed.). Vygotskian approaches to second language research. New Jersey: Ablex, 1994. p. 33-56.

DONATO, R. Vygotskian contributions to understanding the foreign and second language classroom. In: WORLD CONGRESS OF APPLIED LINGUISTICS, 11, 1996, Finland: AILA.

DONATO, R. Sociocultural contributions to understanding the foreign and second language classroom. In: LANTOLF, J. P. (Ed.). Sociocultural theory and second language learning. Hong Kong: Oxford Univ. Press, 2000. p. 27-50.

DONATO, R.; LANTOLF, J. P. The dialogic origins of L2 monitoring. In: BOUTON, L. F.; KACHRU,Y. (Ed.). Pragmatics and language learning. Urbana-Champaign, IL: Division of English as an International Language, 1990. v. 1, p. 83-97.

ELLIS, R. Learning a second language through interaction. Amsterdam: J. Benjamins, 1999. 285 p.

FIGUEIREDO, F. J. Q. Correção com os pares: os efeitos do processo da correção dialogada na aprendizagem da escrita em língua inglesa. 2001. 340 f. Tese (Doutorado em Lingüistica Aplicada). Faculdade de Letras, Universidade Federal de Minas Gerais, Belo Horizonte.

FORMAN, E. A; CAZDEN, C. B. Exploring vygotskian perspectives in education. In: WERTCH, J. V. (Ed.). Culture, communication and cognition. New York: Cambridge Univ. Press, 1985. p. 323-47.

FREEMAN, D. Collaboration: constructing shared understandings in a second language classroom. In: NUNAN, D (Ed.). Collaborative language learning and teaching. Cambridge: Cambridge Univ. Press, 1992. p. 56-80.

GASS, S. M.; VARONIS, E. M. Input, interaction, and second language production. Studies in Second Language Acquisition, v. 16, p. 283-302, 1994.

GASS, S. M.; VARONIS, E. M. Task variation and non-native/non-native negotiation of meaning. In: GASS, S. M.; MADDEN, C. G. (Ed.). Input and second language acquisition. Rowley: Newbury, 1985. p 149-61. 
GASS, S. M.; SELINKER, L. Looking at interlanguage process. In: GASS, S. M.; SELINKER, L. (Ed.). Second language acquisition. Hillsdale: Lawrence Erlbaum Associates, 1994, cap. 6, p. 139-64.

GAUDET, D. et al. La coopération en classe. Montréal: Chenelière/McGrawHill, 1998. 221 p.

KLINGER, J. K.; VAUGHN, S. The helping behaviors of fifth graders while using collaborative strategic reading during ESL content classes. TESOL Quarterly, v. 34, n. 1, p. 69-95, 2000.

KRASHEN, S. D. The input hypothesis. Harlow: Longman, 1983.

LIGHTBOWN, P. M.; SPADA, N. Focus on form and corrective feedback in communicative language teaching. Studies in Second Language Acquisition, v. 12, p. 429-48, 1990.

LONG, M. Native speakers/non-native speakers conversation and the negociation of comprehensible input. Applied Linguistics, v. 4, n. 2, p. 12641, 1983.

LONG, M. The role of the linguistic environment is second language acquisition. In: RITCHIE, W. C.; BATHIA, T. K. (Ed.). Handbook of second language acquisition. New York: Academic Press, 1996. p. 413-68.

LYSTER, R.; RANTA, L. Corrective feedback and learner uptake. Studies in Second Language Acquisition, v. 19, n. 1, p. 37-66, 1997.

PAIVA, V. L. M. O. Diários online na aprendizagem de língua inglesa mediada por computador. In: MARI, H. et al. (Org.). Fundamentos e dimensões da análise do discurso. Belo Horizonte: FALE-UFMG, 1999. p. 359-78.

PICA, T. Research on negociation. Language Learning, v. 44, n. 3, p. 493527, Sept. 1994.

PICA, T. Second language acquisition, social interaction, and the classroom. Applied Linguistics, v. 8, n. 1, p. 3-21, 1987.

PICA, T. et al. Comprehensible output as an outcome of linguistic demands on the learner. Studies in Second Language Acquisition, v. 11, p. 63-90, 1989.

PICA, T. et al. Language learners interaction. TESOL Quarterly, v. 30, n. 1, p. 59-83, Spring. 1996.

PICA, T. et al. Language learning through interaction. Studies in Second Language Acquisition, v. 13, n. 3, p. 343-76, 1991. 
PICA, T.; YOUNG, R.; DOUGHTY, C. The impact of interaction on comprehension. TESOL Quarterly, v. 21, n. 4, p. 737-58, Dec. 1987.

SCHMIDT, R. W.; FROTA, S. N. Developing basic conversational ability in a second language. In: DAY, R. (Ed.). Talking to learn. Rowley: Newbury, 1986. p. 237-326.

SELINKER, L. Interlanguage. International Review of Applied Linguistics, v. 10, p. 209-31, 1972.

STEVAHN, L; BENNETT, B; ROLHEISER, C. L'apprentissage coopératif. Toronto: Educational Connections, 1995. 344 p.

SWAIN, M. Collaborative dialogue: its contribution to second language learning. Trabalho apresentado no The Annual AAAL Conference. Long Beach: California, 1995a.

SWAIN, M. Communicative competence. In: GASS, S.; MADDEN, C. (Ed.). Input in SLA. Rowley: Newbury, 1985. p. 235-53.

SWAIN, M. Focus on form through conscious reflection. In: DOUGHTY, C.; WILLIAMS, J. (Ed.). Focus on form in classroom second language acquisition. Cambridge: Cambridge Univ. Press, 1998. p. 64-82.

SWAIN, M. The output hypothesis and beyond. In: LANTOLF, J. P. (Ed.). Sociocultural theory and second language learning. Hong Kong: Oxford Univ. Press, 2000. cap. 4, p. 97-114.

SWAIN, M. Three functions of output in second language learning. In: COOK, G., SEIDlHOFER, B. (Ed.). Principle \& practice in Applied Linguistics. Oxford: Oxford Univ. Press, 1995b. p. 125-44.

SWAIN, M.; LAPKIN, S. Problems in output and the cognitive process they generate. Applied Linguistics, v. 16, n. 3, p. 371-91, 1995.

SWAIN, M.; LAPKIN, S. Focus on form through collaborative dialogue. In: BYGATE, M.; SKEHAN, P.; SWAIN, M. (Ed.). Researching pedagogic tasks - second language learning, teaching and testing. London: Longman/ Pearson Education, 2001. 258 p.

SWAIN, M.; LAPKIN, S. Interaction and second language learning. The Modern Language Journal, v. 82, n. 3, p. 320-37, 1998.

TUDGE, J. Vygotsky, the zone of proximal development, and peer collaboration. In: MOLL, L. C. (Ed.). Vygotsky and education. New York: Cambridge Univ. Press, 1990. p. 155-72. 
TSUI, A. B. M. Introducing classroom interaction. London: Penguin English, 1995. 122 p.

VAN DEN BRANDEN, K. Effects of negotiation on language learners' output. Language Learning, v. 47, n. 4, p. 589-636, Dec. 1997.

VARONIS, E. M.; GASS, S. Non-native / non-native conversations. Applied Linguistics, v. 6, n. 1, p. 71-90, 1985.

VYGOTSKY, L. S. A formação social da mente. São Paulo: M. Fontes, 1998. $168 \mathrm{p}$.

WELLS, G. Using L1 to master L2. The Canadian Modern Language Review, v. 54, n. 3, p. 343-53, Apr. 1998.

WERTSCH, J. V. Voices of the mind. Cambridge: Harvard Univ. Press, 1991. $165 \mathrm{p}$.

WHITE, J. Getting the learners' attention. In: DOUGHTY, C.; WILLIAMS, J. (Ed.). Focus on form in classroom second language acquisition. Cambridge: Cambridge Univ. Press, 1998. p. 85-113. 